\title{
A Note on Cyclic Duality and Hopf Algebras
}

\author{
M. Khalkhali \\ masoud@uwo.ca \\ Department of Mathematics \\ University of Western Ontario
}

\author{
B. Rangipour* \\ bahram@uvic.ca \\ Department of Mathematics and Statistics \\ University of Victoria
}

November 18, 2018

\begin{abstract}
We show that various cyclic and cocyclic modules attached to Hopf algebras and Hopf modules are related to each other via Connes' duality isomorphism for the cyclic category.
\end{abstract}

\section{Introduction}

A remarkable property of Connes' cyclic category $\Lambda$ is its self duality in the sense that there is a natural isomorphism between $\Lambda$ and its opposite category $\Lambda^{o p}$ [1]. Roughly speaking, the duality functor $\Lambda^{o p} \longrightarrow \Lambda$ acts as identity on objects of $\Lambda$ and exchanges face and degeneracy operators while sending the cyclic operator to its inverse (see Section 2 for a precise formulation). Thus to a cyclic (resp. cocyclic) module one can associate a cocyclic (resp. cyclic) module by applying Connes' duality isomorphism. This notion of duality, called cyclic duality in this paper, should not be confused with the duality obtained by applying the Hom functor, since it is of a very different nature. For example, it is easy to see that the cyclic dual of the cyclic (resp. cocyclic) module of an algebra (resp. coalgebra) is homologically trivial (Lemma 2.3 below).

The goal of this article is to show that the situation is much more interesting for Hopf algebras by showing that various, non-trivial, cyclic and cocyclic modules attached to Hopf algebras and Hopf modules are cyclic duals of each other. Recently we have seen a proliferation of cyclic and cocyclic modules attached to Hopf algebras [5, 11, 9, 8, 6, 7, extending the pioneering work of Connes and Moscovici [3, 4]. Recall that in [6] a cocyclic module $C_{H}^{*}(H, M)$ and a cyclic module $C_{*}^{H}(H, M)$ is defined for any Hopf algebra $H$ and an stable anti-Yetter-Drinfeld $H$-module $M$ (see Section 3 for definitions). For $M=k$, the ground field, these modules reduce to the Connes-Moscovici cocyclic module of an Hopf algebra endowed with a modular pair in involution [3, 4] and to the

${ }^{*}$ PIMS postdoctoral fellow 
cyclic module introduced in 99 11], respectively. It is known that the associated cyclic homology and cohomology theories are non-trivial. In this paper we show that $C_{*}^{H}(H, M)$ is in fact isomorphic, via a non-trivial map, to the cyclic dual of $C_{H}^{*}(H, M)$ (Theorem 3.1).

The cyclic dual of $C_{H}^{*}(H, M)$ appears naturally in the study of the relative cyclic homology of Hopf-Galois extensions [7. Thus our result shows that this theory is a special case of the invariant cyclic homology defined in [6] for general coefficients and in 8 for a restricted class of coefficients.

We would like to thank Nigel Higson for valuable discussions on the subject of this paper. Our thanks go also to our collaborators in [6], Piotr. M. Hajac and Yorck Sommerhäuser.

\section{Duality for cyclic modules}

Let $k$ be a commutative ring with identity and let $k-\bmod$ denote the category of $k$-modules. Recall that a cyclic $k$-module (or a cyclic module for short) is a contravariant functor $\Lambda \rightarrow k-\bmod$, where $\Lambda$ denotes Connes' category [1] (cf. also 2, 10). Equivalently, a cyclic module is given by a sequence $X_{n}, n \geq 0$, of $k$-modules and $k$-linear maps called face, degeneracy and cyclic operators

$$
\delta_{i}: X_{n} \rightarrow X_{n-1}, \quad \sigma_{i}: X_{n} \rightarrow X_{n+1}, \quad \tau_{n}: X_{n} \rightarrow X_{n} \quad 0 \leq i \leq n,
$$

such that $\left(X, \delta_{i}, \sigma_{i}\right)$ is a simplicial module and the following extra relations are satisfied:

$$
\begin{array}{rlrl}
\delta_{i} \tau_{n} & =\tau_{n-1} \delta_{i-1} & 1 \leq i \leq n, \\
\delta_{0} \tau_{n} & =\delta_{n}, & \\
\sigma_{i} \tau_{n} & =\tau_{n-1} \sigma_{i-1} & & 1 \leq i \leq n, \\
\sigma_{0} \tau_{n} & =\tau_{n}^{2} \sigma_{n}, & \\
\tau_{n}^{(n+1)} & =\mathrm{id}_{n} . &
\end{array}
$$

When all relations, except possibly the last one, are satisfied we say that we have a paracyclic module.

A cocyclic module is a functor $\Lambda \longrightarrow k-$ mod. Equivalently, a cocyclic module is given by a sequence of $k$-modules $X^{n}$ and $k$-linear maps called coface, codegeneracy, and cyclic operators:

$$
d_{i}: X^{n} \rightarrow X^{n+1}, \quad s_{i}: X^{n} \rightarrow X^{n-1}, \quad t_{n}: X^{n} \rightarrow X^{n} \quad 0 \leq i \leq n,
$$

such that $\left(X, d_{i}, s_{i}\right)$ is a cosimplicial module and the following extra relations are satisfied:

$$
\begin{array}{rlrl}
t_{n+1} d_{i} & =d_{i-1} t_{n} & & 1 \leq i \leq n, \\
t_{n+1} d_{0} & =d_{n}, & \\
t_{n-1} s_{i} & =s_{i-1} t_{n} & & 1 \leq i \leq n, \\
t_{n-1} s_{0} & =s_{n} t_{n}^{2}, & \\
t_{n}^{(n+1)} & =\mathrm{id}_{n} . &
\end{array}
$$


When all relations, except possibly the last one, are satisfied we say that we have a paracocyclic module.

Let $X=\left(X^{n}, d_{i}, s_{i}, t_{n}\right)$ be a paracocyclic (resp. cocyclic) module where we assume that $t_{n}$ is invertible for all $n \geq 0$. We denote its cyclic dual by $\widehat{X}$. It is defined as follows [1]. We put $\widehat{X}_{n}=X^{n}$, and

$$
\begin{array}{rlr}
\delta_{i} & =s_{i-1}: \widehat{X}_{n} \longrightarrow \widehat{X}_{n-1}, \quad 1 \leq i \leq n, \\
\delta_{0} & =s_{n-1} t_{n}, \\
\sigma_{i} & =d_{i}: \widehat{X}_{n} \longrightarrow \widehat{X}_{n+1}, \\
\tau_{n} & =t_{n}^{-1} .
\end{array}
$$

The following two lemmas are proved in [1]. Both can be checked directly.

Lemma 2.1. $\widehat{X}=\left(\widehat{X}_{n}, \delta_{i}, \sigma_{i}, \tau_{n}\right)$ is a paracyclic module. If $X$ is a cocyclic module, then $\widehat{X}$ is a cyclic module.

Conversely, one can obtain from a paracyclic (resp. cyclic) module a paracocyclic (resp. cocyclic) module as follows. Let $X=\left(X_{n}, \delta_{i}, \sigma_{i}, \tau_{n}\right)$ be a paracyclic module. We denote the cyclic dual of $X$ by $\check{X}$ where $\check{X}^{n}=X_{n}$ and its coface, codegeneracy and cyclic operators are defined by

$$
\begin{array}{rlrl}
d_{i} & =\sigma_{i-1}: \check{X}^{n} \longrightarrow \check{X}^{n+1} & & 1 \leq i \leq n, \\
d_{0} & =\tau_{n} \sigma_{n}, & & \\
s_{i} & =\delta_{i}: \check{X}^{n} \longrightarrow \check{X}^{n-1} & & 0 \leq i \leq n-1, \\
t_{n} & =\tau_{n}^{-1} . &
\end{array}
$$

Lemma 2.2. $\check{X}=\left(\check{X}^{n}, d_{i}, s_{i}, t_{n}\right)$ is a paracocyclic module. If $X$ is a cyclic module, then $\check{X}$ is a cocyclic module.

We give a few examples of cyclic and cocyclic modules that will be used in this paper. The first example is the most fundamental example which motivated the whole theory [1].

1. Let $A$ be an algebra. The cyclic module $A^{\natural}$ is defined by $A_{n}^{\natural}=A^{\otimes(n+1)}, n \geq$ 0 , with face, degeneracy and cyclic operators defined by

$$
\begin{aligned}
\delta_{i}\left(a_{0} \otimes a_{1} \otimes \cdots \otimes a_{n}\right) & =a_{0} \otimes \cdots \otimes a_{i} a_{i+1} \otimes \cdots \otimes a_{n} \\
\delta_{n}\left(a_{0} \otimes a_{1} \otimes \cdots \otimes a_{n}\right) & =a_{n} a_{0} \otimes a_{1} \otimes \cdots \otimes a_{n-1} \\
\sigma_{i}\left(a_{0} \otimes a_{1} \otimes \cdots \otimes a_{n}\right) & =a_{0} \otimes \cdots \otimes a_{i} \otimes 1 \otimes \cdots \otimes a_{n}, \\
\tau_{n}\left(a_{0} \otimes a_{1} \otimes \cdots \otimes a_{n}\right) & =a_{n} \otimes a_{0} \cdots \otimes a_{n-1} .
\end{aligned}
$$

2. Let $C$ be a coalgebra. The cocyclic module $C_{\natural}$ is defined by $C_{\natural}^{n}=$ $C^{\otimes(n+1)}, \quad n \geq 0$, with coface, codegeneracy and cyclic operators given 
by:

$$
\begin{aligned}
d_{i}\left(c_{0} \otimes c_{1} \otimes \cdots \otimes c_{n}\right) & =c_{0} \otimes \cdots \otimes c_{i}^{(1)} \otimes c_{i}^{(2)} \otimes c_{n} \quad 0 \leq i \leq n, \\
d_{n+1}\left(c_{0} \otimes c_{1} \otimes \cdots \otimes c_{n}\right) & =c_{0}^{(2)} \otimes c_{1} \otimes \cdots \otimes c_{n} \otimes c_{0}^{(1)} \\
s_{i}\left(c_{0} \otimes c_{1} \otimes \cdots \otimes c_{n}\right) & =c_{0} \otimes \cdots c_{i} \otimes \varepsilon\left(c_{i+1}\right) \otimes \cdots \otimes c_{n} \quad 0 \leq i \leq n-1, \\
t_{n}\left(c_{0} \otimes c_{1} \otimes \cdots \otimes c_{n}\right) & =c_{1} \otimes c_{2} \otimes \cdots \otimes c_{n} \otimes c_{0},
\end{aligned}
$$

where as usual $\Delta(c)=c^{(1)} \otimes c^{(2)}$ denotes the coproduct of $C$ (Sweedler's notation), and $\varepsilon$ denotes the counit of $C$.

3. Let $H$ be a Hopf algebra, $\delta: H \longrightarrow k$ an algebra map (a character) and $\sigma \in H$ a grouplike element. Following [3] , we say $(\delta, \sigma)$ is a modular pair if $\delta(\sigma)=1$ and a modular pair in involution if, in addition, $\left(\sigma^{-1} \widetilde{S}\right)^{2}=i d_{H}$ where the twisted antipode $\tilde{S}$ is defined by

$$
\widetilde{S}(h)=\delta\left(h^{(1)}\right) S\left(h^{(2)}\right) .
$$

Given $H$ and a modular pair in involution $(\delta, \sigma)$ as above, Connes and Moscovici define a cocyclic module $H_{(\delta, \sigma)}^{\natural}$ as follows. Let $H_{(\delta, \sigma)}^{\natural, 0}=k$ and $H_{(\delta, \sigma)}^{\natural, n}=H^{\otimes n}, n \geq 1$. The coface, codegeneracy and cyclic operators $d_{i}$, $s_{i}, t_{n}$ are defined by

$$
\begin{aligned}
d_{0}\left(h_{1} \otimes \cdots \otimes h_{n}\right) & =1_{\mathcal{H}} \otimes h_{1} \otimes \cdots \otimes h_{n} \\
d_{i}\left(h_{1} \otimes \cdots \otimes h_{n}\right) & =h_{1} \otimes \cdots \otimes \Delta\left(h_{i}\right) \otimes \cdots \otimes h_{n} \text { for } 1 \leq i \leq n \\
d_{n+1}\left(h_{1} \otimes \cdots \otimes h_{n}\right) & =h_{1} \otimes \cdots \otimes h_{n} \otimes \sigma \\
s_{i}\left(h_{1} \otimes \cdots \otimes h_{n}\right) & =h_{1} \otimes \cdots \otimes \epsilon\left(h_{i+1}\right) \otimes \cdots \otimes h_{n} \text { for } 0 \leq i \leq n \\
t_{n}\left(h_{1} \otimes \cdots \otimes h_{n}\right) & =\Delta^{n-1} \widetilde{S}\left(h_{1}\right) \cdot\left(h_{2} \otimes \cdots \otimes h_{n} \otimes \sigma\right) .
\end{aligned}
$$

The cyclic cohomology of this cocyclic module is, by definition, the cyclic cohomology of the Hopf algebra $H$ with respect to $(\delta, \sigma)$.

4. In [9] and, independently, 11, a cyclic module is associated to any Hopf algebra $H$ endowed with a modular pair in involution denoted by $\widetilde{H}_{\natural}^{(\delta, \sigma)}$. We have $\widetilde{H}_{\natural, n}^{(\delta, \sigma)}=H^{\otimes n}$, for $n>0$ and $\widetilde{H}_{\natural, 0}^{(\delta, \sigma)}=k$. Its face, degeneracy, and cyclic operators are as follows:

$$
\begin{aligned}
\delta_{0}\left(h_{1} \otimes h_{2} \otimes \cdots \otimes h_{n}\right)= & \epsilon\left(h_{1}\right) h_{2} \otimes h_{3} \otimes \cdots \otimes h_{n} \\
\delta_{i}\left(h_{1} \otimes h_{2} \otimes \cdots \otimes h_{n}\right)= & h_{1} \otimes h_{2} \otimes \cdots \otimes h_{i} h_{i+1} \otimes \cdots \otimes h_{n} \\
\delta_{n}\left(h_{1} \otimes h_{2} \otimes \cdots \otimes h_{n}\right)= & \delta\left(h_{n}\right) h_{1} \otimes h_{2} \otimes \cdots \otimes h_{n-1} \\
\sigma_{i}\left(h_{1} \otimes h_{2} \otimes \cdots \otimes h_{n}\right)= & h_{1} \otimes h_{2} \cdots \otimes h_{i} \otimes 1 \otimes h_{i+1} \cdots \otimes h_{n} \\
\tau_{n}\left(h_{1} \otimes h_{2} \otimes \cdots \otimes h_{n}\right)= & \delta\left(h_{n}^{(2)}\right) \sigma S\left(h_{1}^{(1)} h_{2}^{(1)} \ldots h_{n-1}^{(1)} h_{n}^{(1)}\right) \otimes \\
& h_{1}^{(2)} \otimes \cdots \otimes h_{n-1}^{(2)} .
\end{aligned}
$$


It is natural to ask what is the relation between the Hochschild and cyclic homology groups of a cyclic module $X$ and the Hochschild and cyclic cohomology groups of its dual cocyclic module $\check{X}$. The following simple lemma answers this question for algebras and coalgebras. In the next section we answer this question for Hopf algebras.

Lemma 2.3. Let $A$ be a unital algebra over a field $k$. Then the Hochschild cohomology of the cocyclic module $\tilde{A}^{\natural}$ is trivial in positive dimensions. Similarly, if $C$ is a coalgebra over $k$, then the Hochschild homology groups of the dual cyclic module $\widehat{C_{\natural}}$ are trivial in positive dimensions.

Proof. Let $\phi$ be a linear functional on $A$ such that $\phi(1)=1$. One can easily check that the following defines a contracting homotopy for the Hochschild complex of $\check{A}$ :

$$
h: A^{\otimes(n+1)} \longrightarrow A^{\otimes n}, \quad h\left(a_{0} \otimes a_{1} \otimes \cdots \otimes a_{n}\right)=\phi\left(a_{0}\right) a_{1} \otimes a_{2} \otimes \cdots \otimes a_{n} .
$$

In the coalgebra case let $c$ be an element of $C$ such that $\epsilon(c)=1$. We define a contracting homotopy for the Hochschild complex of $\hat{C}_{\natural}$ as follows:

$$
s: C^{\otimes n} \longrightarrow C^{\otimes(n+1)}, \quad s\left(c_{0} \otimes c_{1} \otimes \ldots \otimes c_{n-1}\right)=c \otimes c_{0} \otimes c_{1} \otimes \ldots \otimes c_{n-1} .
$$

\section{Cyclic duality and Hopf algebras}

To define a cyclic (co)homology theory with coefficients for Hopf algebras, the module of coefficients must be of a very special type. In [6] the most general allowable Hopf modules of this type are identified and called stable antiYetter-Drinfeld (SAYD) modules. One dimensional SAYD modules correspond exactly to modular pairs in involution. An intermediate case are matched and comatched pairs of 8 . In this section we first recall the notion of an SAYD module over a Hopf algebra and their associated cyclic and cocyclic modules from [6]. We then prove that, quite unexpectedly, these modules are, up to isomorphism, cyclic duals of each other.

If $M$ is a left $H$-comodule we write ${ }_{M} \Delta(m)=m^{(-1)} \otimes m^{(0)}$ to denote its coaction ${ }_{M} \Delta: M \rightarrow H \otimes M$ (Sweedler's notation). Similarly if $M$ is a right $H$-comodule, we write $\Delta_{M}(m)=m^{(0)} \otimes m^{(1)}$ to denote its coaction $\Delta_{M}: M \rightarrow$ $M \otimes H$.

Definition 3.1. Let $H$ be a Hopf algebra with a bijective antipode $S$, and $M$ a module and comodule over $H$. We call $M$ an anti-Yetter-Drinfeld module if the 
action and coaction are compatible in the following sense:

$$
{ }_{M} \Delta(h m)=h^{(1)} m^{(-1)} S^{-1}\left(h^{(3)}\right) \otimes h^{(2)} m^{(0)},
$$

if $M$ is a left module and a left comodule ;

$$
\Delta_{M}(h m)=h^{(2)} m^{(0)} \otimes h^{(3)} m^{(1)} S\left(h^{(1)}\right),
$$

if $M$ is a left module and a right comodule ;

$$
{ }_{M} \Delta(m h)=S\left(h^{(3)}\right) m^{(-1)} h^{(1)} \otimes m^{(0)} h^{(2)},
$$

if $M$ is a right module and a left comodule ;

$$
\Delta_{M}(m h)=m^{(0)} h^{(2)} \otimes S^{-1}\left(h^{(1)}\right) m^{(1)} h^{(3)},
$$

if $M$ is a right module and a right comodule.

In the first case we say $M$ is stable if $m^{(-1)} m^{(0)}=m$ for all $m \in M$ (similar definitions apply in other cases).

Let $M$ be an SAYD H-module of the second type in the above definition (left module and right comodule). Let $C_{n}^{a l g}(H, M):=M \otimes H^{\otimes(n+1)}$. It is shown in [6] that the following operators define a paracyclic module structure on $\left\{C_{n}^{a l g}(H, M)\right\}_{n}$ :

$$
\begin{aligned}
\delta_{i}\left(m \otimes h_{0} \otimes \cdots \otimes h_{n}\right) & =m \otimes h_{0} \otimes \cdots \otimes h_{i} h_{i+1} \otimes \cdots \otimes h_{n}, \\
\delta_{n}\left(m \otimes h_{0} \otimes \cdots \otimes h_{n}\right) & =h_{n}^{(1)} m \otimes h_{n}^{(2)} h_{0} \otimes \cdots \otimes h_{n-1}, \\
\sigma_{i}\left(m \otimes h_{0} \otimes \cdots \otimes h_{n}\right) & =m \otimes h_{0} \otimes \cdots \otimes h_{i} \otimes 1 \otimes h_{i+1} \otimes \ldots h_{n}, \\
\tau\left(m \otimes h_{0} \otimes \cdots \otimes h_{n}\right) & =h_{n}^{(1)} m \otimes h_{n}^{(2)} \otimes h_{0} \otimes \cdots \otimes h_{n-1} .
\end{aligned}
$$

It is shown in [6] that the above operators restrict to the subcomplex $C_{n}^{H}(H, M):=$ $M \square_{H} H^{\otimes(n+1)}$ of invariant chains on $H$ with coefficients in $M$ and define a cyclic module that we denote it by $C_{*}^{H}(H, M)$. Here $\square$ denotes the cotensor product. We recall that, in general, the cotensor product $M \square_{H} N$ of a right $H$-comodule $M$ and a left $H$-comodule $N$ is defined as the kernel of the map

$$
\Delta_{M} \otimes 1-1 \otimes_{N} \Delta: M \otimes N \longrightarrow M \otimes H \otimes N .
$$

The cyclic homology of $H$ with coefficients in $M$ is by definition the cyclic homology of this module. For $M=k$, we are reduced to the cyclic module $\widetilde{H}_{\natural}^{(\delta, \sigma)}$ defined in [11] and, independently, [9].

With $H$ and $M$ as above, let $C_{\text {coalg }}^{n}(H, M):=H^{\otimes(n+1)} \otimes M$. Endowed with the following operators, $\left\{C_{\text {coalg }}^{n}(H, M)\right\}^{n}$ is a paracocyclic module [6]:

$$
\begin{aligned}
d_{i}\left(h_{0} \otimes \cdots \otimes h_{n} \otimes m\right) & =h_{0} \otimes \cdots \otimes h_{i}^{(1)} \otimes h_{i}^{(2)} \otimes h_{i+1} \otimes \cdots \otimes h_{n} \otimes m, \\
d_{n+1}\left(h_{0} \otimes \cdots \otimes h_{n} \otimes m\right) & =h_{0}^{(2)} \otimes h_{1} \otimes \cdots \otimes h_{n} \otimes h_{0}^{(1)} S^{-1}\left(m^{(1)}\right) \otimes m^{(2)}, \\
s_{i}\left(h_{0} \otimes \cdots \otimes h_{n} \otimes m\right) & =h_{0} \otimes \cdots \otimes \epsilon\left(h_{i}\right) \otimes \cdots \otimes h_{n} \otimes m, \\
t_{n}\left(h_{0} \otimes \cdots \otimes h_{n} \otimes m\right) & =h_{1} \otimes h_{2} \cdots \otimes h_{n} \otimes h_{0} S^{-1}\left(m^{(1)}\right) \otimes m^{(0)} .
\end{aligned}
$$

To define the cyclic cohomology of $H$ with coefficients in $M$ we consider the quotient complex $C_{H}^{n}(H, M):=H^{\otimes(n+1)} \otimes_{H} M$ of invariant cochains on $H$ with 
coefficients in $M$. It is shown in [6] that $C_{H}^{*}(H, M)$ is in fact a cocyclic module. For $M=k$, we obtain the Connes-Moscovici cocyclic module $H_{(\delta, \sigma)}^{\natural}$.

A quick look at the above two modules show that there is no apparent cyclic duality relationship between $C_{H}^{*}(H, M)$ and $C_{*}^{H}(H, M)$. In the following, however, we will show, via a very non-trivial map, that they are indeed cyclic dual of each other. Consider the dual paracyclic module $K_{*}(H, M):=\widehat{C}_{\text {coalg }}^{*}(H, M)$. Using our formulas in Section 2, its simplicial and cyclic operators are given by:

$$
\begin{aligned}
\delta_{i}\left(h_{0} \otimes h_{1} \otimes \cdots \otimes h_{n} \otimes m\right) & =h_{0} \otimes \cdots \otimes \epsilon\left(h_{i}\right) \otimes \cdots \otimes h_{n} \otimes m, \\
\sigma_{i}\left(h_{0} \otimes h_{1} \otimes \cdots \otimes h_{n} \otimes m\right) & =h_{0} \otimes h_{i}^{(1)} \otimes h_{i}^{(2)} \otimes \cdots \otimes h_{n} \otimes m, \\
\tau\left(h_{0} \otimes h_{1} \otimes \cdots \otimes h_{n} \otimes m\right) & =h_{n} m^{(1)} \otimes h_{o} \otimes \cdots \otimes h_{n-1} \otimes m^{(0)} .
\end{aligned}
$$

Proposition 3.1. The following map defines a morphism of paracyclic modules $\theta: K_{*}(H, M) \longrightarrow C_{*}^{a l g}(H, M)$,

$$
\begin{aligned}
\theta\left(h_{0} \otimes h_{1} \otimes \cdots \otimes h_{n} \otimes m\right)= & h_{n}^{(2)} m^{(0)} \otimes h_{n}^{(3)} m^{(1)} S\left(h_{0}^{(1)}\right) \otimes h_{0}^{(2)} S\left(h_{1}^{(1)}\right) \otimes h_{1}^{(2)} S\left(h_{2}^{(1)}\right) \otimes \\
& \cdots \otimes h_{n-1}^{(2)} S\left(h_{n}^{(1)}\right) .
\end{aligned}
$$

Proof. To prove that $\theta$ is a cyclic map one needs to show that $\theta \delta_{i}=\delta_{i} \theta, \theta \sigma_{i}=$ $\sigma_{i} \theta$ for $1 \leq i \leq n$, and $\theta \tau=\tau \theta$. Here we just check the latter identity and leave the rest to the reader.

$$
\begin{aligned}
& \theta \tau\left(h_{0} \otimes h_{1} \otimes \ldots \otimes h_{n} \otimes m\right)=\theta\left(h_{n} m^{(1)} \otimes h_{o} \otimes \ldots \otimes h_{n-1} \otimes m^{(0)}\right) \\
& =h_{n-2}^{(2)} m^{(0)} \otimes h_{n-1}^{(3)} m^{(1)} S\left(h_{n}^{(1)} m^{(2)}\right) \otimes h^{(2)} m^{(3)} S\left(h_{0}^{(1)}\right) \otimes \ldots \otimes h_{n-2}^{(2)} S\left(h_{n-1}^{(1)}\right) \\
& =h_{n-2}^{(2)} m^{(0)} \otimes h_{n-1}^{(3)} S\left(h_{n}^{(1)}\right) \otimes h_{n}^{(2)} m^{(1)} S\left(h_{0}^{(1)}\right) \otimes \ldots \otimes h_{n-2}^{(2)} S\left(h_{n-1}^{(1)}\right) \\
& =h_{n-2}^{(2)} S\left(h_{n}^{(2)}\right) h_{n}^{(3)} m^{(0)} \otimes h_{n-1}^{(3)} S\left(h_{n}^{(1)}\right) \otimes h_{n}^{(4)} m^{(1)} S\left(h_{0}^{(1)}\right) \otimes \ldots \otimes h_{n-2}^{(2)} S\left(h_{n-1}^{(1)}\right) \\
& =\left(h_{n-2}^{(2)} S\left(h_{n}^{(1)}\right)^{(1)} h_{n}^{(3)} m^{(0)} \otimes\left(h_{n-1}^{(2)} S\left(h_{n}^{(1)}\right)\right)^{(2)} \otimes h_{n}^{(3)} m^{(1)} S\left(h_{0}^{(1)}\right) \otimes \ldots \otimes h_{n-2}^{(2)} S\left(h_{n-1}^{(1)}\right)\right. \\
& =\tau \theta\left(h_{0} \otimes h_{1} \otimes \ldots \otimes h_{n} \otimes m\right) .
\end{aligned}
$$

The map $\theta$ is neither injective nor surjective. We can however show that it descends to the relevant spaces of invariants on both sides and gives an isomorphism of cyclic modules:

Lemma 3.1. The map $\theta$ lands in $M \square_{H} H^{\otimes(n+1)}$ and descends to the quotients $H^{\otimes(n+1)} \otimes_{H} M$ for each $n$.

Proof. To prove the second part we show that for all $g, h_{0}, h_{1}, \ldots h_{n} \in H$ and $m \in M$ we have $\theta\left(\left(h_{0} \otimes h_{1} \otimes \ldots \otimes h_{n}\right) \cdot g \otimes m\right)=\theta\left(h_{0} \otimes h_{1} \otimes \ldots \otimes h_{n} \otimes g m\right)$. 
Indeed:

$$
\begin{aligned}
& \theta\left(\left(h_{0} \otimes h_{1} \otimes \ldots \otimes h_{n}\right) \cdot g \otimes m\right)=\theta\left(h_{0} g^{(1)} \otimes h_{1} g^{(2)} \otimes \ldots \otimes h_{n} g^{(n+1)} \otimes m\right) \\
& =\left(h_{n} g^{(n+1)}\right)^{(2)} m^{(0)} \otimes\left(h_{n} g^{(n+1)}\right)^{(3)} m^{(1)} S\left(\left(h_{0} g^{(1)}\right)^{(1)}\right) \otimes\left(h_{0} g^{(1)}\right)^{(2)} S\left(\left(h_{1} g^{(2)}\right)^{(1)}\right) \otimes \\
& \left(h_{1} g^{(2)}\right)^{(2)} S\left(\left(h_{2} g^{(3)}\right)^{(1)}\right) \otimes \cdots \otimes\left(h_{n-1} g^{(n)}\right)^{(2)} S\left(\left(h_{n} g^{(n+1)}\right)^{(1)}\right) \\
& =h_{n}^{(2)} g^{(2 n+2)} m^{(0)} \otimes h_{n}^{(3)} g^{(2 n+3)} m^{(1)} S\left(g^{(1)}\right) S\left(h_{0}^{(1)}\right) \otimes h_{0}^{(2)} g^{(2)} S\left(g^{(3)}\right) S\left(h_{1}^{(1)}\right) \otimes \\
& h_{1}^{(2)} g^{(4)} S\left(g^{(5)}\right) S\left(h_{2}^{(1)}\right) \otimes \cdots \otimes\left(h_{n-1}^{(2)} g^{(2 n)} S\left(g^{(2 n+1)}\right) S\left(h_{n}^{(1)}\right)\right. \\
& =h_{n}^{(2)} g^{(2)} m^{(0)} \otimes h_{n}^{(3)} g^{(3)} m^{(1)} S\left(g^{(1)}\right) \otimes h_{0}^{(2)} S\left(h_{1}^{(1)}\right) \otimes \ldots \otimes h_{n-1}^{(2)} S\left(h_{n}^{(1)}\right) .
\end{aligned}
$$

On the other hand, by using the stable anti-Yetter-Drinfeld module property of $M$, one has:

$$
\begin{aligned}
& \theta\left(h_{0} \otimes h_{1} \otimes \ldots \otimes h_{n} \otimes g m\right)= \\
& h_{n}^{(2)}(g m)^{(0)} \otimes h_{n}^{(3)}(g m)^{(1)} S\left(h_{0}^{(1)}\right) \otimes h_{0}^{(2)} S\left(h_{1}^{(1)}\right) \otimes \ldots \otimes h_{n-1}^{(2)} S\left(h_{n}^{(1)}\right) \\
& h_{n}^{(2)} g^{(2)} m^{(0)} \otimes h_{n}^{(3)} g^{(3)} m^{(1)} S\left(g^{(1)}\right) \otimes h_{0}^{(2)} S\left(h_{1}^{(1)}\right) \otimes \ldots \otimes h_{n-1}^{(2)} S\left(h_{n}^{(1)}\right) .
\end{aligned}
$$

Now we prove that the image of $\theta$ is in the cotensor product space $M \square_{H} H^{\otimes(n+1)}$. That is, $\left(\Delta_{M} \otimes i d_{H \otimes(n+1)}\right) \circ \theta=\left(i d_{M} \otimes_{H \otimes(n+1)} \Delta\right) \circ \theta$.

$\Delta_{M} \otimes i d_{H \otimes(n+1)} \circ \theta\left(h_{0} \otimes h_{1} \otimes \ldots \otimes h_{n} \otimes m\right)$

$$
\begin{aligned}
& =\Delta_{M} \otimes i d_{H^{(n+1)}}\left(h_{n}^{(2)} m^{(0)} \otimes h_{n}^{(3)} m^{(1)} S\left(h_{0}^{(1)}\right) \otimes h_{0}^{(2)} S\left(h_{1}^{(1)}\right) \otimes h_{1}^{(2)} S\left(h_{2}^{(1)}\right) \otimes \cdots\right. \\
& \left.\otimes h_{n-1}^{(2)} S\left(h_{n}^{(1)}\right)\right) \\
& =\left(h_{n}^{(2)} m^{(0)}\right)^{(0)} \otimes\left(h_{n}^{(2)} m^{(0)}\right)^{(1)} \otimes h_{n}^{(3)} m^{(1)} S\left(h_{0}^{(1)}\right) \otimes h_{0}^{(2)} S\left(h_{1}^{(1)}\right) \otimes h_{1}^{(2)} S\left(h_{2}^{(1)}\right) \otimes \cdots \\
& \left.\otimes h_{n-1}^{(2)} S\left(h_{n}^{(1)}\right)\right) \\
& \left.=h_{n}^{(3)} m^{(0)} \otimes h_{n}^{(4)} m^{(1)} S\left(h_{n}^{(2)}\right) \otimes h_{n}^{(5)} m^{(2)} S\left(h_{0}^{(1)}\right) \otimes h_{0}^{(2)} S\left(h_{1}^{(1)}\right) \otimes \cdots \otimes h_{n-1}^{(2)} S\left(h_{n}^{(1)}\right)\right)
\end{aligned}
$$

On the other hand, we have:

$$
\begin{aligned}
& i d_{M} \otimes_{H \otimes(n+1)} \Delta \circ \theta\left(h_{0} \otimes h_{1} \otimes \ldots \otimes h_{n} \otimes m\right)= \\
& =h_{n}^{(2)} m^{(0)} \otimes\left(h_{n}^{(3)} m^{(1)} S\left(h_{0}^{(1)}\right)\right)^{(1)}\left(h_{0}^{(2)} S\left(h_{1}^{(1)}\right)\right)^{(1)} \ldots\left(h_{n-1}^{(2)} S\left(h_{n}^{(1)}\right)\right)^{(1)} \otimes \\
& \left(h_{n}^{(3)} m^{(1)} S\left(h_{0}^{(1)}\right)\right)^{(2)} \otimes\left(h_{0}^{(2)} S\left(h_{1}^{(1)}\right)\right)^{(2)} \otimes \ldots \otimes\left(h_{n-1}^{(2)} S\left(h_{n}^{(1)}\right)\right)^{(2)} \\
& \left.=h_{n}^{(3)} m^{(0)} \otimes h_{n}^{(4)} m^{(1)} S\left(h_{n}^{(2)}\right) \otimes h_{n}^{(5)} m^{(2)} S\left(h_{0}^{(1)}\right) \otimes h_{0}^{(2)} S\left(h_{1}^{(1)}\right) \otimes \cdots \otimes h_{n-1}^{(2)} S\left(h_{n}^{(1)}\right)\right) .
\end{aligned}
$$

Theorem 3.1. The induced map $\theta$ is an isomorphism of cyclic modules

$$
\theta: K_{*}^{H}(H, M) \longrightarrow C_{*}^{H}(H, M) \text {. }
$$

Proof. At first we identify $H^{\otimes(n+1)} \otimes_{H} M$ with $H^{\otimes n} \otimes M$. Consider the map $\phi: H^{\otimes(n+1)} \otimes M \rightarrow H^{\otimes n} \otimes M$, $\phi\left(h_{0} \otimes h_{1} \otimes \ldots \otimes h_{n} \otimes m\right)=h_{0} S\left(h_{n}^{(n)}\right) \otimes h_{1} S\left(h_{n}^{(2)}\right) \otimes \ldots \otimes h_{n-1} S\left(h_{n}^{(1)}\right) \otimes h_{n}^{(n+1)} m$. 
One can see that this map is $H$-bilinear. So it induces a map $\bar{\phi}: H^{\otimes(n+1)} \otimes_{H}$ $M \rightarrow H^{\otimes n} \otimes M$. It can easily be checked that the following map is the two-sided inverse of $\bar{\phi}$ :

$$
\begin{aligned}
& \psi: H^{\otimes n} \otimes M \rightarrow H^{\otimes(n+1)} \otimes_{H} M \\
& \psi\left(h_{1} \otimes h_{2} \otimes \ldots \otimes h_{n} \otimes m\right)=h_{1} \otimes h_{2} \otimes \ldots \otimes h_{n} \otimes 1 \otimes_{H} m .
\end{aligned}
$$

Next we identify $M \square_{H} H^{\otimes(n+1)}$ with $M \otimes H^{\otimes n}$. Consider the maps $\phi^{\prime}$ : $M \square_{H} H^{\otimes(n+1)} \longrightarrow M \otimes H^{\otimes n}$,

$$
\phi^{\prime}\left(m \otimes h_{0} \otimes \ldots \otimes h_{n}\right)=m \otimes \epsilon\left(h_{0}\right) h_{1} \otimes h_{2} \otimes \ldots \otimes h_{n}
$$

and $\psi^{\prime}: M \otimes H^{\otimes n} \longrightarrow M \square_{H} H^{\otimes(n+1)}$

$\psi^{\prime}\left(m \otimes h_{1} \otimes h_{2} \otimes \ldots \otimes h_{n}\right)=m^{(0)} \otimes m^{(1)} S\left(h_{1}^{(1)} h_{2}^{(1)} \ldots h_{n}^{(1)}\right) \otimes h_{1}^{(1)} \otimes h_{2}^{(1)} \otimes \ldots \otimes h_{n}^{(1)}$.

One can check that $\phi^{\prime}$ and $\psi^{\prime}$ are inverse to one another.

After the above identifications, the map induced by $\theta$, denoted $\bar{\theta}$, on $H^{\otimes(n+1)} \otimes_{H}$ $M$, has the following formula:

$$
\begin{aligned}
& \bar{\theta}: H^{\otimes n} \otimes M \longrightarrow M \otimes H^{\otimes n}, \\
& \bar{\theta}\left(h_{1} \otimes h_{2} \otimes \ldots \otimes h_{n} \otimes m\right)=m^{(0)} \otimes m^{(1)} S\left(h_{0}^{(1)}\right) \otimes h_{0}^{(2)} S\left(h_{1}^{(1)}\right) \otimes \ldots \\
& \otimes h_{n-2}^{(2)} S\left(h_{n-1}^{(1)}\right) \otimes h_{n-1}^{(2)} .
\end{aligned}
$$

One can directly check that $\bar{\theta}$ is an isomorphism and its inverse is given by

$$
\begin{aligned}
& \gamma: M \otimes H^{\otimes n} \longrightarrow H^{\otimes n} \otimes M \\
& \gamma\left(m \otimes h_{1} \otimes h_{2} \otimes \ldots \otimes h_{n}\right)=S\left(m^{(1)}\right) h_{0} h_{1}^{(1)} \ldots h_{n-1}^{(1)} \otimes h_{1}^{(2)} \ldots h_{n-1}^{(2)} \otimes \ldots \\
& \otimes h_{n-2}^{(n-1)} h_{n_{2}}^{(n-1)} \otimes h_{n-1}^{(n)} \otimes m^{(0)} .
\end{aligned}
$$

The cyclic module $K_{*}^{H}(H, M)$ is used by Jara and Stefan in their study of relative cyclic homology of Hopf-Galois extensions 77. Note that what we call an stable anti-Yetter-Drinfeld module in the present paper (and in [6]), is called a modular crossed module in [7. It follows from Theorem 3.1 above that Theorem 4.13 in [7] is a consequence of Theorem 3.1 in [6] (by choosing $A=H$ ). For the same reason, Theorem 5.2 in [7] follows from Theorem 3.22 in our paper [8] (for special classes of SAYD modules called matched pairs, but the same proof works in general).

In the remainder of this paper we briefly look into a different type of duality, i.e. the Hom-functor duality, between the cyclic module $C_{*}^{H}(H, M)$ and the cocyclic module $C_{H}^{*}(G, N)$. Recall that a Hopf pairing between Hopf algebras $G$ and $H$ is a bilinear map $<,>: H \otimes G \longrightarrow k$ that satisfies the following relations for all $h, h_{1}, h_{2}$ in $H$ and $g, g_{1}, g_{2}$ in $G$ :

$$
<h_{1} h_{2}, g>=<h_{1}, g^{(1)}><h_{2}, g^{(2)}>, \quad<h, g_{1} g_{2}>=<h^{(1)}, g_{1}><h^{(2)}, g_{2}>,
$$




$$
<h, 1>=\varepsilon(h), \quad<1, g>=\varepsilon(g) .
$$

In addition let $M \in{ }_{H} \mathcal{M}^{H}$ be a left $H$-moodule and a right $H$-comodule, and $N \in{ }^{G} \mathcal{M}_{G}$ be a left $G$-comodule and a right $G$-module, both satisfying the SAYD-module conditions. To complete the set up for having a pairing between Hopf cyclic cohomologies we need a pairing between $M$ and $N$, denoted here by $<,>: M \otimes N \rightarrow k$, such that for all $m \in M, n \in N, h \in H, g \in G$, the following conditions are satisfied:

$<h m, n>=<h, n^{(-1)}><m, n^{(0)}>, \quad<m, n g>=<m^{(0)}, n><m^{(1)}, g>$.

Consider the map $M \otimes H^{\otimes n} \longrightarrow \operatorname{Hom}\left(N \otimes G^{\otimes n}, k\right)$ defined by

$$
\left(m \otimes h_{1} \otimes \cdots \otimes h_{n}\right)\left(n \otimes g_{1} \otimes \cdots \otimes g_{n}\right)=<m, n>\prod_{i=1}^{i=n}<h_{i}, g_{i}>.
$$

Proposition 3.2. The above map defines a morphism of cocyclic modules

$$
C_{\text {coalg }}^{*}(H, M) \longrightarrow H \text { om }_{k}\left(C_{*}^{\text {alg }}(G, N), k\right) .
$$

If $H$ is finite dimensional over a field $k$ and $G=H^{*}$ is the dual Hopf algebra, then it is easy to see that the above map, for $M=N=k$ and the natural pairing between $H$ and $H^{*}$, is an isomorphism of cocyclic modules. Thus the Connes-Moscovici cyclic cohomology of a finite dimensional Hopf algebra is isomorphic to the cyclic cohomology in the sense of 9 11] of $G=H^{*}$. In the infinite dimensional case, however, the induced map on cohomology can be trivial, even for a non-degenerate pairing. For example, let $H=\operatorname{Rep}(\mathbf{G})$ be the Hopf algebra of representable functions on a compact Lie group $\mathbf{G}$ and $G=U(\mathfrak{g})$ the enveloping algebra of the Lie algebra $\mathfrak{g}$ of $\mathbf{G}$. The canonical pairing $H \otimes G \rightarrow \mathbb{C}$ defined by

$$
<f, X_{1} \otimes \cdots \otimes X_{n}>=\left.\frac{d}{d t} f\left(e^{t X_{1}} \ldots e^{t X_{n}}\right)\right|_{t=0}
$$

is non-degenerate. Then while the Connes-Moscovici cyclic cohomology of $H$ is trivial, the cyclic cohomology of $G$ in the sense of 91 11] is non-trivial and in fact is isomorphic to the Lie algebra cohomology of $\mathfrak{g}$ (see 9 for a proof of both statements).

In Lemma 2.3 we saw that cyclic duals of cyclic modules of algebras and coalgebras are both homologically trivial. It follows from Theorem 3.1 that this need not be true for cyclic modules of Hopf algebras. In fact for $H=U(\mathfrak{g})$ and the modular pair $(\delta, \sigma)=(\varepsilon, 1)$, the periodic cyclic cohomology and homology are computed in [3] and [9] respectively. They are both isomorphic to the Lie algebra homology of $\mathfrak{g}$.

\section{References}

[1] A. Connes, Cohomologie cyclique et foncteurs $\operatorname{Ext}^{n}$. C. R. Acad. Sci. Paris Sr. I Math. 296 (1983), no. 23, 953-958. 
[2] A. Connes, Noncommutative geometry. Academic Press, Inc., San Diego, CA, 1994.

[3] A. Connes and H. Moscovici, Hopf algebras, Cyclic Cohomology and the transverse index theorem, Comm. Math. Phys. 198 (1998), no. 1, 199-246.

[4] A. Connes and H. Moscovici, Cyclic cohomology and Hopf algebra symmetry. Conference Mosh Flato 1999 (Dijon). Lett. Math. Phys. 52 (2000), no. 1, $1-28$.

[5] M. Crainic, Cyclic cohomology of Hopf algebras, J. Pure Appl. Algebra 166 (2002), no. 1-2, 29-66.

[6] P.M. Hajac, M. Khalkhali, B. Rangipour, and Y. Sommerhäuser, Hopf-cyclic homology and cohomology with coefficients. arXiv:math.KT/0306288

[7] P. Jara, and D. Stefan, Cyclic homology of Hopf Galois extensions and Hopf algebras, arXiv:math.KT/0307099

[8] M. Khalkhali, and B. Rangipour, Invariant cyclic homology. $K$-Theory 28(2), 183-205, 2003.

[9] M. Khalkhali, and B. Rangipour, A new cyclic module for Hopf algebras. $K$-Theory 27(2), 111-131, 2002.

[10] J. L. Loday, Cyclic Homology. Springer-Verlag, (1992).

[11] R. Taillefer, Cyclic Homology of Hopf Algebras. K-Theory 24 (2001), no. $1,69-85$. 